\title{
On the Electroporation Thresholds of Lipid Bilayers: Molecular Dynamics Simulation Investigations
}

\author{
Andraž Polak • Daniel Bonhenry • François Dehez • \\ Peter Kramar • Damijan Miklavčič • \\ Mounir Tarek
}

Received: 4 February 2013/Accepted: 31 May 2013/Published online: 19 June 2013

(C) Springer Science+Business Media New York 2013

\begin{abstract}
Electroporation relates to the cascade of events that follows the application of high electric fields and that leads to cell membrane permeabilization. Despite a wide range of applications, little is known about the electroporation threshold, which varies with membrane lipid composition. Here, using molecular dynamics simulations, we studied the response of dipalmitoyl-phosphatidylcholine, diphytanoyl-phosphocholine-ester and diphytanoylphosphocholine-ether lipid bilayers to an applied electric field. Comparing between lipids with acyl chains and methyl branched chains and between lipids with ether and ester linkages, which change drastically the membrane dipole potential, we found that in both cases the electroporation threshold differed substantially. We show, for the first time, that the electroporation threshold of a lipid bilayer depends not only on the "electrical" properties of
\end{abstract}

\footnotetext{
A. Polak $\cdot$ P. Kramar · D. Miklavčič

Faculty of Electrical Engineering, University of Ljubljana,

Tržaška cesta 25, 1000 Ljubljana, Slovenia

e-mail: andraz.polak@fe.uni-lj.si

P. Kramar

e-mail: peter.kramar@fe.uni-lj.si

D. Miklavčič

e-mail: damijan.miklavcic@fe.uni-lj.si

D. Bonhenry · F. Dehez · M. Tarek $(\square)$

CNRS, Unité Mixte de Recherche 7565, Université de Lorraine,

Campus Science Grignard, Vandoeuvre-lés-Nancy, BP 239,

54506 Nancy Cedex, France

e-mail: mounir.tarek@univ-lorraine.fr

D. Bonhenry

e-mail: Daniel.bonhenry@univ-lorraine.fr

F. Dehez

e-mail: francois.dehez@univ-lorraine.fr
}

the membrane, i.e., its dipole potential, but also on the properties of its component hydrophobic tails.

Keywords Capacitance - Electroporation threshold . Membrane dipole potential $\cdot$ DPPC $\cdot$ DPhPC

\section{Introduction}

Electroporation relates to a phenomenon in which cell membranes are permeabilized when they are exposed to high electric fields (Neumann and Rosenheck 1972). Several studies based on molecular dynamics (MD) simulations have shown that pores are formed when lipid bilayers, considered as simple models of cell membranes, are subject to such conditions. First, defects (water wires) penetrate the hydrophobic core of the bilayers; then, these expand to form water-filled pores spanning the whole bilayer, which are later stabilized by reorganization of lipid molecules (Levine and Vernier 2010; Tarek 2005; Tieleman 2004). Cell electroporation is used in several fields, like biology, biotechnology and medicine (Haberl et al. 2013). Electroporation is considered reversible if cells recover their initial state after the electric field is switched off; electroporation is considered irreversible if it leads to cell death. These two processes are used in electrochemotherapy (Sersa et al. 2008), transdermal drug delivery (Denet et al. 2004), gene therapy (Daud et al. 2008; Breton et al. 2012), water cleaning (Vernhes et al. 2002), food processing (Toepfl et al. 2007) and tissue ablation (Davalos et al. 2005).

Electroporation is also a potentially valuable method used to release drugs from uploaded synthetic liposomes (Napotnik et al. 2010), when these reach selected intracellular target areas (Elbayoumi and Torchilin 2010). Obviously, the electroporation of synthetic liposomes 
depends on the structure of their constitutive lipids and on their response to an applied electric field (Portet et al. 2009). Accordingly, for efficient use of the protocol, the structure of the membrane and its behavior under an electric field have to be known.

Archaea membranes have high stability in harsh environments (Benvegnu et al. 2004; Ulrih et al. 2009) and as such are very good candidates for use as liposomes for drug delivery (Hanford and Peeples 2002; Ulrih et al. 2009). Their stability is probably due to several factors that directly relate to the peculiar chemical structure of the lipids of which they are composed. Compared to simple phosphatidylcholine lipids, archaeal lipids have a special headgroup formed of sugar moieties but also methyl branches in the lipid tails and ether linkages instead of ester linkages between the headgroup and the carbonyl region (Ulrih et al. 2009). Comparison between dipalmitoylphosphatidylcholine (DPPC)—, diphytanoyl-phosphocholine-ester (DPhPC-ester) - and diphytanoyl-phosphocholine-ether (DPhPC-ether)—based bilayers show, for instance, that branched chains increase the stability and decrease the permeability (Shinoda et al. 2003, 2004b) of the membrane, which are related to the slower conformational motion of the lipid tails. The difference in ester and ether linkage affects, on the other hand, the headgroup hydration and the membrane electrostatic potential. DPhPC-ester bilayers, for instance, have a dipole potential two times larger than DPhPC-ether bilayers (Shinoda et al. 2004a). We may therefore expect a direct effect on the stability of such bilayers under electrical stress, i.e., exposure to an electric field. Similar studies have reported effects on 1,2-dilauroyl-sn-phosphatidylcholine (DLPC), DPPC, palmitoyl-2-oleoyl-phosphatidylcholine (POPC), 1,2-dioleoyl-sn-phosphatidylcholine (DOPC), single-component lipid membranes (Ziegler and Vernier 2008) and POPC lipid membranes with incorporated cholesterol (Fernández et al. 2010). In both studies transmembrane voltages were induced by exposing the membranes to an external electric field. The results showed that different lipid tails have an effect on the electroporation threshold. In addition, the presence of increasing cholesterol amounts in simple lipid membranes was shown to increase the electroporation threshold (Needham and Hochmuth 1989).

In the present article, we propose specifically to evaluate the influence of branched lipid tails and headgroup linkage (ester and ether) on the electroporation of bilayers composed of archaeal-type lipids using atomistic MD simulations.

\section{Materials and Methods}

We studied three bilayers composed of DPPC, DPhPCester or DPhPC-ether lipids (Fig. 1). DPPC was modeled using the all-atom CHARMM 36 force field (Klauda et al. 2010). DPhPC-ester and DPhPC-ether are not described in this force field topology database; the former was therefore built by adding four methyl groups to each tail of DPPC lipid, while the latter was subsequently built by changing the ester bond to an ether bond in the DPhPC-ester molecules. The force field parameters of ether linkage were adopted from Shinoda et al. (2004a).

The MD simulations presented here were carried out using the program NAMD (Kalé et al. 1999). The systems were examined in the NPT (constant number of atoms, pressure and temperature) and NVT (constant number of atoms, volume and temperature) ensembles employing
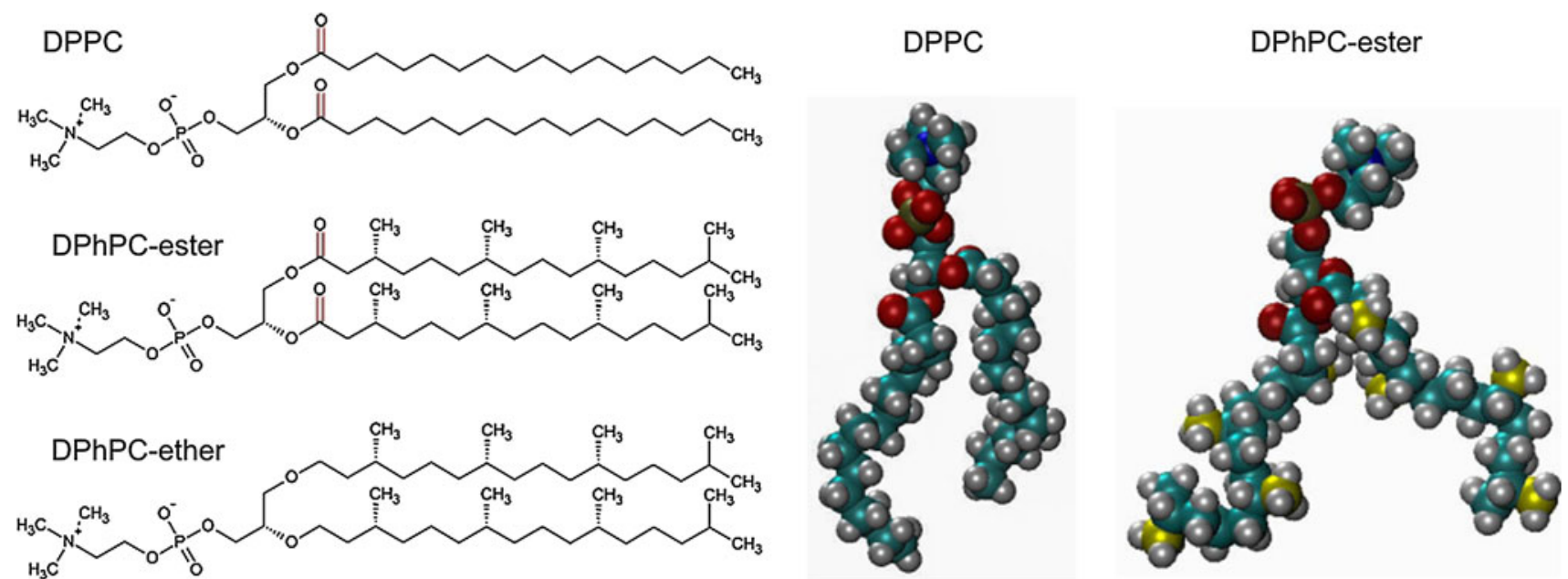

due to similarity to the $\mathrm{DPhPC}$-ester). The atoms in the models are color-labeled $(\mathrm{O}$, red $; \mathrm{C}$, cyan and yellow; $\mathrm{H}$, gray; $\mathrm{N}$, blue $; \mathrm{P}$, brown $)$

Fig. 1 Left Sketches of the studied lipid molecules (DPPC, DPhPCester and DPhPC-ether). Right MD models of the studied lipid molecules (DPPC and DPhPC-ester, the DPhPC-ether is not shown 
Langevin dynamics and the Langevin piston method. The time step for integrating the equations of motion was set at 2.0 fs. Short- and long-range forces were calculated every one and two time steps, respectively. Bonds between hydrogen and heavy atoms were constrained to their equilibrium value. Long-range, electrostatic forces were taken into account using the particle mash Ewald (PME) approach (Darden et al. 1993; Essmann et al. 1995).

All systems were considered at a $\sim 0.45 \mathrm{M} \mathrm{KCl}$ solution and contained enough lipids to form large patches. The DPPC bilayer was hence composed of 256 lipid molecules, 20,174 water molecules, 153 potassium $\left(\mathrm{K}^{+}\right)$ions and 153 chloride $\left(\mathrm{Cl}^{-}\right)$ions. The DPhPC-ester and DPhPC-ether membranes were composed each of $256 \mathrm{DPhPC}$ molecules, 25,888 water molecules, $196 \mathrm{~K}^{+}$ions and $196 \mathrm{Cl}^{-}$ions. The numbers of ions and water molecules differ among systems, but the chosen salt molar concentration was the same for all systems. For further calculation, systems without ions were also considered and constructed by removing the ions from the equilibrated DPhPC-ester/ether systems with ions. These were mainly used to compare our results to existing data in order to check the validity of the force field and the protocols used.

Equilibrations of the bilayers were performed at constant pressure (1 atm.) and constant temperatures (50 and $25{ }^{\circ} \mathrm{C}$ ) for tens of nanoseconds. The last $15 \mathrm{~ns}$ of each simulation had a stable area per lipid; therefore, these intervals were assumed to be equilibrated runs and were used for the analyses.

The electrostatic potential profiles along the membrane normal was derived from MD simulations using Poisson's equation and expressed as the double integral of the molecular charge density distributions, $\rho(z): \Phi(z)=$ $-\varepsilon_{0}^{-1} \iint \rho\left(z^{\prime \prime}\right) \mathrm{d} z^{\prime \prime} \mathrm{d} z^{\prime}, z$ being the position of the charge in the direction along the normal to the bilayer.

The capacitance of the simulated membranes was calculated using the charge imbalance method (Delemotte et al. 2008; Sachs et al. 2004). Selected configurations from the equilibrated NPT runs were used to set new systems, where the simulation box size was extended in the direction perpendicular to the membrane to create an air-water interface. For these runs, the temperature was maintained at $50{ }^{\circ} \mathrm{C}$ and the volume was maintained constant. Systems with charge imbalances of $0,2,4,6$ and 8e were simulated for over $10 \mathrm{~ns}$ each. The last $5 \mathrm{~ns}$ of simulation were analyzed for electrostatic potential distribution, from which the transmembrane voltages $\left(U_{\mathrm{t}}\right)$ were calculated. For all simulations, $U_{\mathrm{t}}$ was found in a linear correlation with $q$, the charge imbalance normalized to the membrane area. Accordingly, the capacitance of the bilayers was estimated as $c=q / U_{\mathrm{t}}$.

Electroporation of the lipid bilayers was induced by applying high transmembrane voltages created by means of the charge imbalance method. All MD simulations were run at several voltages. The electroporation threshold ( $\left.U_{\mathrm{EPthres}}\right)$ is reported as the interval between the highest $U_{\mathrm{t}}$ at which lipid bilayers are not electroporated in the 100-ns timescale and the lowest $U_{\mathrm{t}}$ at which pores are created in the membrane.

\section{Results}

The time evolutions of the surface area per lipid for DPPC, DPhPC-ester and DPhPC-ether bilayers in $0.45 \mathrm{M} \mathrm{KCl}$ at $50{ }^{\circ} \mathrm{C}$ and in no-salt solutions at 50 and $25^{\circ} \mathrm{C}$ show that the membranes were well equilibrated within few tens of nanoseconds (data not shown). The average areas per lipid for both DPhPC-ester and DPhPC-ether (respectively, $80.1 \pm 0.6$ and $\left.74.6 \pm 0.7 \AA^{2}\right)$ are much higher than for the DPPC bilayer $\left(60.0 \pm 0.9 \AA^{2}\right)$ (Table 1). Note here that the average DPPC area/molecule value found here is similar to that reported by Klauda et al. (2010) $\left(59.1 \pm 0.4 \AA^{2}\right)$ and smaller than the experimentally reported one, $63.0 \pm 1.0 \AA^{2}$ (Kucerka et al. 2008), with no ions. The areas per molecule for the DPhPC-ester and DPhPC-ether bilayers slightly change in the absence of salt and as the temperature is further lowered to $25^{\circ} \mathrm{C}$ (Table 1). At $25{ }^{\circ} \mathrm{C}$ the values found here for DPhPC-ester

Table 1 Properties of the equilibrated DPPC, DPhPC-ester and DPhPC-ether bilayers from MD simulations

\begin{tabular}{lllll}
\hline Bilayers & Buffer $(\mathrm{M} \mathrm{KCl})$ & $T\left({ }^{\circ} \mathrm{C}\right)$ & Area per lipid $\left(\AA^{2}\right)$ & Dipole potential $(\mathrm{V})$ \\
\hline DPPC & 0.45 & 50 & $60.0 \pm 0.9\left(59.1,{ }^{\mathrm{a}} 63.0^{\mathrm{c}}\right)$ & $0.70\left(0.7,,^{\mathrm{a}} 0.24^{\mathrm{f}, \mathrm{g}}\right)$ \\
DPhPC-ester & 0.45 & 50 & $80.1 \pm 0.6$ & 0.62 \\
& - & 50 & $79.9 \pm 0.6$ & 0.58 \\
DPhPC-ether & 0.45 & 25 & $78,6 \pm 0.6\left(77.7,{ }^{\mathrm{b}} 76^{\mathrm{d}}\right)$ & $0.62\left(1.00,{ }^{\mathrm{b}} 0.51^{\mathrm{e}}\right)$ \\
& - & 50 & $74.6 \pm 0.7$ & 0.36 \\
& & 50 & $75.7 \pm 0.6$ & 0.38 \\
\end{tabular}

Simulation data: ${ }^{\text {a }}$ Klauda et al. (2010) and ${ }^{\text {b }}$ Shinoda et al. (2004a). Experimental data: ${ }^{\mathrm{c}}$ Kucerka et al. (2008), ${ }^{\mathrm{d}}$ Wu et al. (1995), ${ }^{\mathrm{e}}$ Wang et al. (2006), ${ }^{\mathrm{f}}$ Gawrisch et al. (1992) and ${ }^{\mathrm{g}}$ Peterson et al. (2002) 
Fig. 2 Left Total density profiles of DPPC, DPhPC-ester and $\mathrm{DPhPC}$-ether bilayers in $0.45 \mathrm{M} \mathrm{KCl}$ and at $50{ }^{\circ} \mathrm{C}$. Right Water and lipid tail density profiles of DPPC, DPhPC-ester and $\mathrm{DPhPC}$-ether bilayers in $0.45 \mathrm{M} \mathrm{KCl}$ and $50{ }^{\circ} \mathrm{C}$

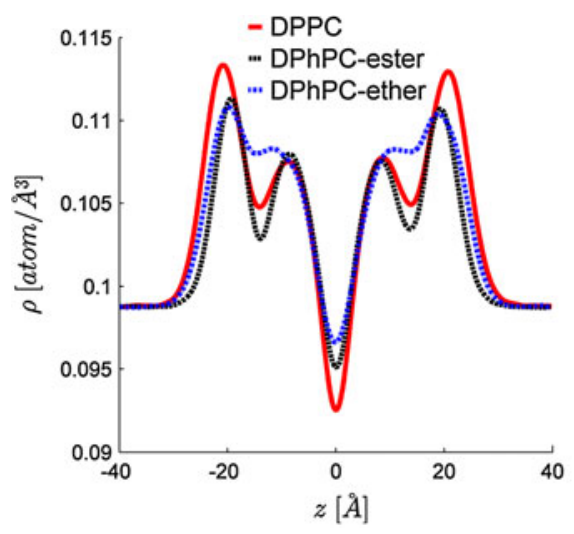

and DPhPC-ether $\left(78.6 \pm 0.6\right.$ and $\left.73.6 \pm 0.4 \AA^{2}\right)$ match quite nicely those reported by others (Shinoda et al. 2004a) (77.7 and $74.1 \AA^{2}$ ). Finally, as a comparison, the experimentally determined area per molecule for DPhPC-ester is $76 \AA^{2}$ (Wu et al. 1995), which provides some confidence in the force field and protocol used here. The density profiles across the DPPC, DPhPC-ester and DPhPC-ether bilayers in $\mathrm{KCl}$ solution are reported in Fig. 2.

The electrostatic potential profiles across the DPPC, DPhPC-ester and DPhPC-ether bilayers were estimated from the distribution of charges in the systems using the Poisson equation. The presence of ions in the systems does not modify the electrostatic potential profiles much (data not shown). Also, the lipid bilayers at temperatures of 50 and $25{ }^{\circ} \mathrm{C}$ have similar overall electrostatic potential profiles; therefore, these profiles are shown only for systems with ions (Fig. 3). Because of the similarity of their headgroups, DPPC and DPhPC-ester electrostatic profiles are very close. There is, in contrast, a large difference between the profiles of ester and ether DPhPCs. The socalled membrane dipole potentials (measure of the difference between voltages between the outside and the

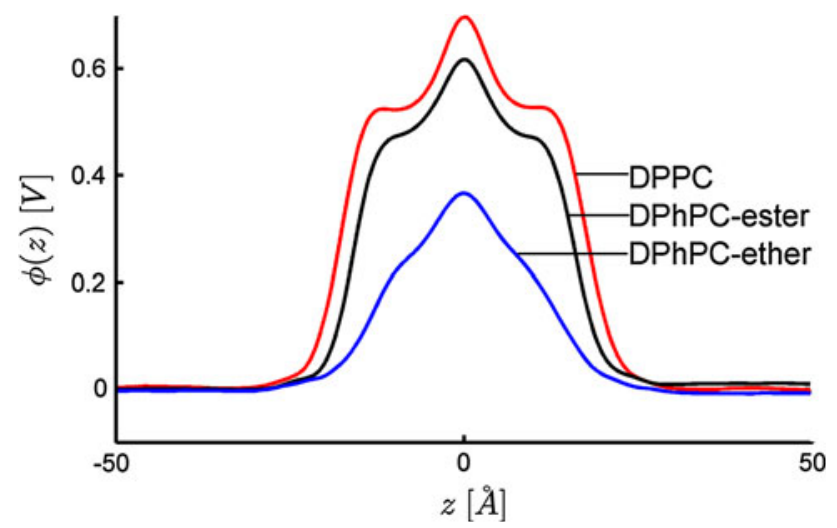

Fig. 3 Electrostatic potential profiles of DPPC, DPhPC-ester and DPhPC-ether bilayers in $0.45 \mathrm{M} \mathrm{KCl}$ and $50{ }^{\circ} \mathrm{C}$. Average estimated dipole potentials of DPPC, DPhPC-ester and DPhPC-ether bilayers are $0.70,0.62$ and $0.36 \mathrm{~V}$, respectively hydrophobic core of the membrane) of DPPC, DPhPC-ester and DPhPC-ether measured from the corresponding electrostatic profiles are, respectively, $0.70,0.62$ and $0.36 \mathrm{~V}$.

For DPPC, there is a large difference between the experimental dipole potential values [using the charge relaxation method $0.23 \mathrm{~V}$ (Gawrisch et al. 1992) and $0.24 \mathrm{~V}$ (Peterson et al. 2002)] and those derived from our MD simulations, which are consistent with the $0.7 \mathrm{~V}$ found using a similar force field (Klauda et al. 2010). However, for DPhPC, the simulation data are in good agreement with those derived using cryo-EM, where the dipole potentials for DPhPC-ester and DPhPC-ether were, respectively, 0.51 and $0.26 \mathrm{~V}$ (Wang et al. 2006).

Similar analyses were performed for the systems with no salt concentration and at lower temperature. These changes, as previously mentioned, had little effect on the shape of the electrostatic profiles; and accordingly, the magnitudes of the dipole potentials were quite similar (Table 1).

Lipid bilayers behave as capacitors (Delemotte et al. 2008). Hence, by imposing a charge imbalance across the lipid bilayers (DPPC, DPhPC-ester and DPhPC-ether), one may create a transmembrane voltage across these bilayers. These transmembrane voltages are found to be proportional to the charge imbalance normalized to the lipid bilayer area (Fig. 4). By imposing charge imbalances $(0,2,4,6$ and 8e) to the modeled systems at $0.45 \mathrm{M} \mathrm{KCl}$, we have estimated their capacitances. There is only a mild difference $(0.94$, 0.93 and $0.90 \mu \mathrm{F} \mathrm{cm}^{-2}$ ) between the values found, respectively, for DPPC, DPhPC-ester and DPhPC-ether. Again, there is a discrepancy between these values and those determined experimentally: $\sim 0.38 \mu \mathrm{F} \mathrm{cm} \mathrm{cm}^{-2}$ of DPPC (Antonov et al. 1990) and DPhPC-ester bilayers found by others to range from 0.5 to $0.6 \mu \mathrm{F} \mathrm{cm}{ }^{-2}$ (Ridi et al. 1998). Consistent with previous studies on POPC (Kramar et al. 2012), all capacitance values determined from atomistic simulations were much higher than those determined experimentally.

Having hence characterized the electrostatic properties of the three bilayers, we performed additional simulations 

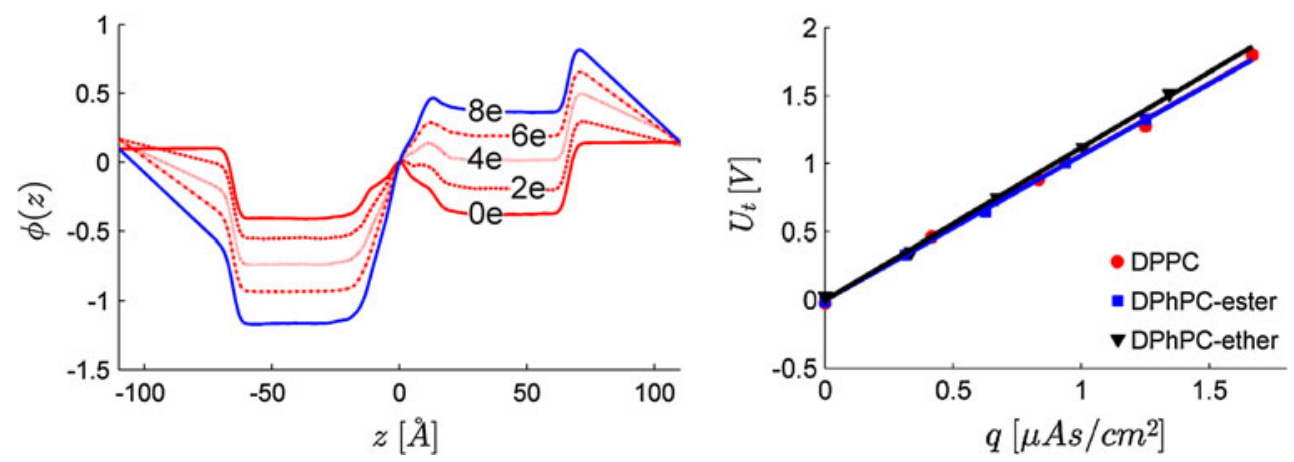

Fig. 4 Left Electrostatic potential profiles across the DPhPC-ether bilayers for simulations performed at different net charge imbalances $Q(0-8 \mathrm{e})$ between the upper and lower electrolytes. Right Transmembrane potentials across the DPPC, DPhPC-ester and DPhPC-

in order to determine $U_{\mathrm{EPthres}}$, i.e., their electroporation thresholds. Simulations of DPPC, DPhPC-ester and DPhPC-ether lipid bilayers at several applied transmembrane voltages were performed. The times the hydrophobic pore is created and the first ion goes through the pore are shown in Table 2. In all simulations, above the threshold, the pores are created in the 30-ns timescale. For voltages where no pore was created, the simulations were extended for more than $60 \mathrm{~ns}$. Hence, electroporation of DPPC, DPhPC-ester and DPhPC-ether lipid bilayers did not occurr at, respectively, $\sim 1.8,2.3$ and $3.0 \mathrm{~V}$ for extended-length simulations. On the contrary, electroporation (creation of pores) of the lipid bilayers occurred in less than $30 \mathrm{~ns}$ for DPPC, DPhPC-ester and DPhPC-ether bilayers at, respectively, $\sim 2.3,3.0$ and $3.7 \mathrm{~V}$.

\section{Discussion}

In this MD simulations study, we compared DPPC-, DPhPC-ester- and DPhPC-ether-based lipid bilayers characteristics and responses to electrical stress. Analyses of the simulations of the bilayers showed that the electrostatic potentials across the DPPC, DPhPC-ester and DPhPC-ether bilayers are independent of the surrounding solvent salt concentration and only slightly change for temperatures varying from 25 to $50{ }^{\circ} \mathrm{C}$. These electrostatic potentials depend, however, on the membrane's lipid composition, in particular, the nature of the tails and to a much greater extent the nature of the linkages (ester and ether) from headgroup to tail.

We investigated the stability of DPPC, DPhPC-ester and DPhPC-ether bilayers under an electric stress. Comparison of the electroporation thresholds obtained from MD simulations under conditions mimicking the effect of lowmagnitude millisecond electric pulses (Delemotte and Tarek 2012) suggests that both the nature of the lipid tails

ether bilayers as a function of the charge imbalance $(Q)$ normalized to the area of membranes. The slopes represent linear fits to the data that permit estimates of bilayer capacitance

and the type of linkages (ester and ether) have an effect. Changing the lipid from DPPC to DPhPC, i.e., addition of methyl groups in the hydrophobic tails, stabilizes the lipid bilayer and increases the electroporation threshold, $U_{\text {EPth- }}$ res. Changing the ester linkage with an ether when going from DPhPC-ester to DPhPC-ether stabilizes even more the bilayer and increases further the $U_{\mathrm{EPthres}}$. Hence, the $U_{\text {EPthres }}$ values for DPPC, DPhPC-ester and DPhPC-ether bilayers were between 1.8 and $2.2 \mathrm{~V}$, between 2.3 and $2.7 \mathrm{~V}$ and between 3.0 and $3.4 \mathrm{~V}$, respectively. Note that fine-tuning these ranges can be achieved if the systems modeled were much larger lipid patches. The ranges nonetheless indicate here clearly a threshold increase.

These "absolute values" of the $U_{\text {EPthres }}$ increase are probably overestimated, as are so far all the electroporation thresholds determined from MD simulations. It is not yet clear to us what are the reasons behind such large discrepancies between electroporation thresholds found in simulations and those determined experimentally. Considering the electrical properties of the membranes, the dipole potential of DPPC-based membranes is in agreement with other MD simulations but is much higher than experimental results measured by invasive methods (Table 1). On the other hand, the membrane dipole potentials of DPhPCester and -ether membranes are only slightly higher than those determined experimentally using noninvasive methods (e.g., cryo-EM). Quite interestingly, the ratio between membrane dipole potentials of DPhPC-ester and -ether determined from MD simulations $(\sim 2)$ is the same as that found in experiments. We also considered the link to the lipid tail properties via the permittivity of the membrane, $\varepsilon$, and therefore the capacitance, $C$, of the bilayer: $C=\varepsilon A / d$. The capacitances of the membranes measured using the charge imbalance method for all three lipids are around $0.9 \mu \mathrm{F} \mathrm{cm}^{-2}$, which is about two times higher than those obtained experimentally. A similar discrepancy was obtained for POPC bilayers (Kramar et al. 2012). 
Table 2 Characteristics of DPPC, DPhPC-ester and DPhPC-ether lipid bilayers under various transmembrane voltages created by a net charge imbalance

\begin{tabular}{|c|c|c|c|c|c|}
\hline Bilayers & $\begin{array}{l}\text { Charge } \\
\text { imbalance (e) }\end{array}$ & $\begin{array}{l}\text { Transmembrane } \\
\text { voltage }(\mathrm{V})\end{array}$ & $\begin{array}{l}\text { Simulation } \\
\text { (ns) }\end{array}$ & $\begin{array}{l}\text { Water wire } \\
\text { formed (ns) }\end{array}$ & $\begin{array}{l}\text { First ion } \\
\text { through (ns) }\end{array}$ \\
\hline \multirow[t]{6}{*}{ DPPC } & 8 & 1.8 & 63 & - & - \\
\hline & 10 & 2.2 & 14 & 2.5 & 2.7 \\
\hline & & & 18 & 16.5 & 16.9 \\
\hline & & & 10 & - & - \\
\hline & & & 10 & - & - \\
\hline & 12 & 2.6 & 10 & 1.5 & 1.8 \\
\hline \multirow[t]{8}{*}{ DPhPC-ester } & 14 & 2.3 & 67 & - & - \\
\hline & 16 & 2.7 & 38 & 12.5 & 13.3 \\
\hline & & & 37 & - & - \\
\hline & 18 & 3.0 & 27 & 12.0 & 12.6 \\
\hline & 20 & 3.3 & 10 & 4.1 & 5.2 \\
\hline & & & 10 & 1.9 & 2.9 \\
\hline & & & 10 & 2.0 & 2.4 \\
\hline & & & 10 & 4.5 & 5.2 \\
\hline \multirow[t]{9}{*}{ DPhPC-ether } & 16 & 3.0 & 66 & - & - \\
\hline & 18 & 3.4 & 51 & 26.8 & 28.0 \\
\hline & 20 & 3.7 & 26 & 15.9 & 17.5 \\
\hline & 22 & 4.1 & 10 & 6.1 & 7.0 \\
\hline & & & 10 & 6.5 & 6.7 \\
\hline & & & 10 & 3.5 & 4.0 \\
\hline & & & 10 & - & - \\
\hline & 24 & 4.5 & 10 & 2.0 & 2.7 \\
\hline & 26 & 4.9 & 4 & 2.3 & 2.7 \\
\hline
\end{tabular}

We performed a few analyses seeking to relate the reason for changes in the electroporation threshold between the lipid bilayers to their structural and physical properties. The profiles of the three bilayers indicate only a mild change in the density profiles between the three components and, therefore, do not provide strong evidence of variability that can be directly correlated with the changes in $U_{\text {EPthres }}$. The correspondence between $U_{\text {EPthres }}$ and the lipid bilayer capacitance is also weak since the force fields used do not provide for a large change in the lipid bilayer capacitances, while there is a significant change in their $U_{\text {EPthres }}$.

We have also estimated the local pressure profiles (Lindahl and Edholm 2000) along $z$, the bilayer normal, at various system configurations. The pressure profiles may be calculated from simulations as $p(z)=$ $\frac{1}{\Delta V}\left[\sum_{i} m_{i} v_{i} \otimes v_{i}-\sum_{i<j} F_{i j} \otimes r_{i j} f\left(z, z_{i}, z_{j}\right)\right]$, where $p(z)$ is the local pressure tensor in the slab centered on the coordinate $z$, the sum over the kinetic term running over all atoms in the slab and $f\left(z, z_{i}, z_{j}\right)$ a weighting function. All calculations were performed on the fly (Kalé et al. 1999) from the simulations performed at constant temperature and constant pressure. In a lipid bilayer, pressure profiles arise due to the amphipathic nature of the lipids composing it: the hydrophilic headgroups are squeezed together to prevent exposure of the hydrophobic tails to the solvent leading to a negative lateral pressure, while the attractive dispersion forces and entropic repulsion between the lipid tails result mainly in a positive lateral pressure.

Comparison of the pressure profiles of DPPC and DPhPC-ester (Fig. 5) showed again only a mild increase for the branched chain lipid bilayer in the upper region of the hydrophobic tails. It is unlikely that such an increase in the local membrane lateral tension affects greatly the $U_{\text {EPthres }}$.

Shinoda et al. (2004b) performed an extensive study of water permeability in both membranes that may provide a rationale for the increased electroporation threshold as we go from DPPC to DPhPC. Indeed, the authors show from analyses of local diffusion coefficients that water molecules have considerably reduced mobility in the DPhPC membrane interior compared with the DPPC membrane interior. As a result of reduced water diffusion in the branch chained membrane, the water permeability of the DPhPC bilayer was less than that of the DPPC bilayer by about $30 \%$. As such permeability or diffusion toward the 


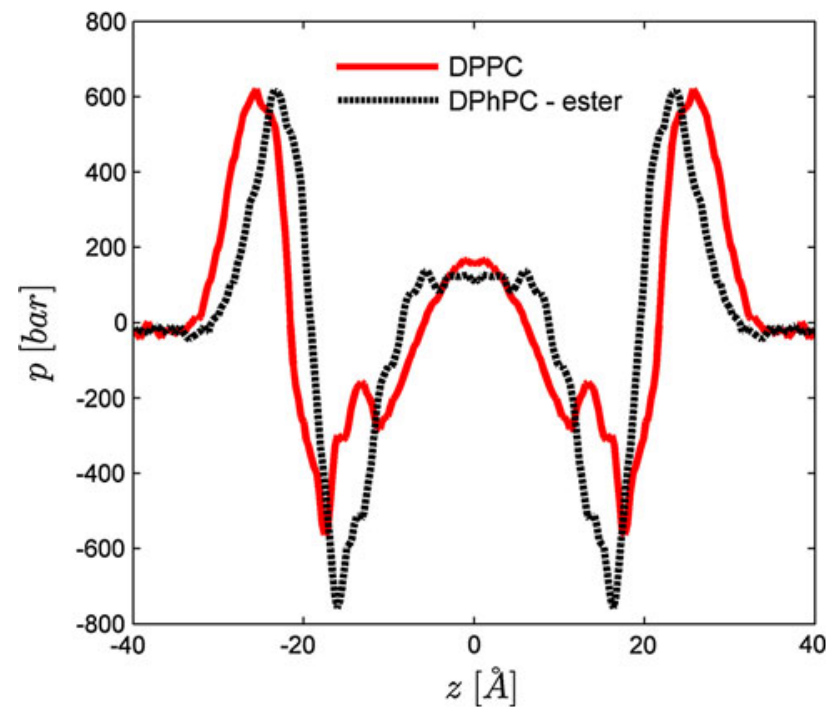

Fig. 5 Lateral pressure profile of DPPC and DPhPC-ester bilayers in $0.45 \mathrm{M} \mathrm{KCl}$ and at $50{ }^{\circ} \mathrm{C}$

interior of the membrane is the very initial key step in electroporation (Delemotte and Tarek 2012; Tockman et al. 2013), we expect that this has a direct impact on $U_{\text {EPthres }}$.

Evidently, more studies comparing different lipid bilayers are needed in order to understand and determine the link between the electroporation threshold and the specificities of the lipid components of a membrane. The present results, however, show clearly that the electroporation threshold of a lipid bilayer depends not only on the "electrical" properties of the membrane, i.e., its dipole potential, but also on the properties of its components' hydrophobic tails.

Acknowledgments This work was in part supported by the Slovenian Research Agency. Research was conducted in the scope of the EBAM European Associated Laboratory. The article is a result of the networking efforts of COST Action TD1104. Part of the calculations and the finalization of the article was performed during the ShortTerm Scientific Mission (Grant 070113-021794, to A. P.). Simulations were performed using HPC resources from GENCI-CINES (Grant 2012-076434). The authors thank the ANR Intcell program (ANR-10-BLAN-096).

\section{References}

Antonov VF, Smirnova EY, Shevchenko EV (1990) Electric field increases the phase transition temperature in the bilayer membrane of phosphatidic acid. Chem Phys Lipids 52:251-257

Benvegnu T, Brard M, Plusquellec D (2004) Archaeabacteria bipolar lipid analogues: structure, synthesis and lyotropic properties. Curr Opin Colloid Interface Sci 8:469-479

Breton M, Delemotte L, Silve A, Mir LM, Tarek M (2012) Nanosecond pulsed electric field driven transport of siRNA molecules through lipid membranes: an experimental and computational study. J Am Chem Soc 134:13938-13941
Darden T, York D, Pedersen L (1993) Particle mesh Ewald: an $\mathrm{N} \cdot \log (\mathrm{N})$ method for Ewald sums in large systems. J Chem Phys 98:10089

Daud AI, DeConti RC, Andrews S et al (2008) Phase I trial of interleukin-12 plasmid electroporation in patients with metastatic melanoma. J Clin Oncol 26:5896-5903

Davalos RV, Mir LM, Rubinsky B (2005) Tissue ablation with irreversible electroporation. Ann Biomed Eng 33:223-231

Delemotte L, Tarek M (2012) Molecular dynamics simulations of lipid membrane electroporation. J Membr Biol 245:531-543

Delemotte L, Dehez F, Treptow W, Tarek M (2008) Modeling membranes under a transmembrane potential. J Phys Chem B 112:5547-5550

Denet A-R, Vanbever R, Préat V (2004) Skin electroporation for transdermal and topical delivery. Adv Drug Deliv Rev 56:659-674

Elbayoumi TA, Torchilin VP (2010) Current trends in liposome research. Methods Mol Biol 605:1-27

Essmann U, Perera L, Berkowitz ML et al (1995) A smooth particle mesh Ewald method. J Chem Phys 103:8577

Fernández ML, Marshall G, Sagués F, Reigada R (2010) Structural and kinetic molecular dynamics study of electroporation in cholesterol-containing bilayers. J Phys Chem B 114:6855-6865

Gawrisch K, Ruston D, Zimmerberg J et al (1992) Membrane dipole potentials, hydration forces, and the ordering of water at membrane surfaces. Biophys J 61:1213-1223

Haberl S, Miklavcic D, Sersa G et al (2013) Cell membrane electroporation-part 2. The applications. IEEE Electr Insulation Mag 29:29-37

Hanford MJ, Peeples TL (2002) Archaeal tetraether lipids: unique structures and applications. Appl Biochem Biotechnol 97:45-62

Kalé L, Skeel R, Bhandarkar M et al (1999) NAMD2: greater scalability for parallel molecular dynamics. J Comput Phys 151:283-312

Klauda JB, Venable RM, Freites JA et al (2010) Update of the CHARMM all-atom additive force field for lipids: validation on six lipid types. J Phys Chem B 114:7830-7843

Kramar P, Delemotte L, Lebar AM et al (2012) Molecular-level characterization of lipid membrane electroporation using linearly rising current. J Membr Biol 245:651-659

Kucerka N, Nagle JF, Sachs JN et al (2008) Lipid bilayer structure determined by the simultaneous analysis of neutron and X-ray scattering data. Biophys J 95:2356-2367

Levine ZA, Vernier PT (2010) Life cycle of an electropore: fielddependent and field-independent steps in pore creation and annihilation. J Membr Biol 236:27-36

Lindahl E, Edholm O (2000) Spatial and energetic-entropic decomposition of surface tension in lipid bilayers from molecular dynamics simulations. J Chem Phys 113:3882

Napotnik TB, Rebersek M, Kotnik T et al (2010) Electropermeabilization of endocytotic vesicles in B16 F1 mouse melanoma cells. Med Biol Eng Comput 48:407-413

Needham D, Hochmuth RM (1989) Electro-mechanical permeabilization of lipid vesicles. Role of membrane tension and compressibility. Biophys J 55:1001-1009

Neumann E, Rosenheck K (1972) Permeability changes induced by electric impulses in vesicular membranes. J Membr Biol 10:279-290

Peterson U, Mannock DA, Lewis RNA et al (2002) Origin of membrane dipole potential: contribution of the phospholipid fatty acid chains. Chem Phys Lipids 117:19-27

Portet T, Camps I, Febrer F, Escoffre J-M et al (2009) Visualization of membrane loss during the shrinkage of giant vesicles under electropulsation. Biophys J 96:4109-4121

Ridi A, Scalas E, Robello M, Gliozzi A (1998) Linear response of a fluctuating lipid bilayer. Thin Solid Films 327-329:796-799 
Sachs JN, Crozier PS, Woolf TB (2004) Atomistic simulations of biologically realistic transmembrane potential gradients. J Chem Phys 121:10847-10851

Sersa G, Miklavcic D, Cemazar M et al (2008) Electrochemotherapy in treatment of tumours. Eur J Surg Oncol 34:232-240

Shinoda W, Mikami M, Baba T, Hato M (2003) Molecular dynamics study on the effect of chain branching on the physical properties of lipid bilayers: structural stability. J Phys Chem B 107:14030-14035

Shinoda K, Shinoda W, Baba T, Mikami M (2004a) Comparative molecular dynamics study of ether- and ester-linked phospholipid bilayers. J Chem Phys 121:9648-9654

Shinoda W, Mikami M, Baba T, Hato M (2004b) Molecular dynamics study on the effects of chain branching on the physical properties of lipid bilayers: 2. Permeability. J Phys Chem B 108:9346-9356

Tarek M (2005) Membrane electroporation: a molecular dynamics simulation. Biophys J 88:4045-4053

Tieleman DP (2004) The molecular basis of electroporation. BMC Biochem 5:10

Tockman M, Lee JH, Levine ZA, Ho M-C, Colvin ME, Vernier PT (2013) Electric field-driven water dipoles: nanoscale architecture of electroporation. PLoS One 8(4):e61111
Toepfl S, Heinz V, Knorr D (2007) High intensity pulsed electric fields applied for food preservation. Chem Eng Process 46:537-546

Ulrih NP, Gmajner D, Raspor P (2009) Structural and physicochemical properties of polar lipids from thermophilic Archaea. Appl Microbiol Biotechnol 84:249-260

Vernhes M, Benichou A, Pernin P et al (2002) Elimination of freeliving amoebae in fresh water with pulsed electric fields. Water Res 36:3429-3438

Wang L, Bose PS, Sigworth FJ (2006) Using cryo-EM to measure the dipole potential of a lipid membrane. Proc Natl Acad Sci USA 103:18528-18533

Wu Y, He K, Ludtke SJ, Huang HW (1995) X-ray diffraction study of lipid bilayer membranes interacting with amphiphilic helical peptides: diphytanoyl phosphatidylcholine with alamethicin at low concentrations. Biophys J 68:2361-2369

Ziegler MJ, Vernier PT (2008) Interface water dynamics and porating electric fields for phospholipid bilayers. J Phys Chem B 112:13588-13596 